Corrigendum

\title{
Corrigendum to "Health-Related Quality of Life and Sleep Quality after 12 Months of Treatment in Nonsevere Obstructive Sleep Apnea: A Randomized Clinical Trial with Continuous Positive Airway Pressure and Mandibular Advancement Splints"
}

\author{
Lars M. Berg $\mathbb{D}^{1},{ }^{1}$ Torun K. S. Ankjell, ${ }^{2,3}$ Yi-Qian Sun, ${ }^{4,5}$ Tordis A. Trovik, ${ }^{6}$ \\ Oddveig G. Rikardsen, ${ }^{2,3}$ Anders Sjögren, ${ }^{1}$ Ketil Moen, ${ }^{7}$ Sølve Hellem, ${ }^{8}$ \\ and Vegard Bugten 9,10 \\ ${ }^{1}$ Department of Clinical Dentistry, Faculty of Health Sciences, UiT the Arctic University of Norway, Tromsø, Norway \\ ${ }^{2}$ ENT Department, University Hospital in Northern Norway, Tromsø, Norway \\ ${ }^{3}$ Department of Clinical Medicine, Faculty of Health Sciences, UiT the Arctic University of Norway, Tromsø, Norway \\ ${ }^{4}$ Center for Oral Health Services and Research, Mid-Norway (TkMidt), Trondheim, Norway \\ ${ }^{5}$ Department of Clinical and Molecular Medicine, Faculty of Medicine and Health Sciences, \\ NTNU Norwegian University of Science and Technology, Trondheim, Norway \\ ${ }^{6}$ Department of Community Medicine, Faculty of Health Sciences, UiT the Arctic University of Norway, Tromsø, Norway \\ ${ }^{7}$ ENT Department, Section for Oral and Maxillofacial Surgery, Arendal Hospital, Arendal, Norway \\ ${ }^{8}$ Department of Clinical Dentistry, Faculty of Medicine, University of Bergen, Bergen, Norway \\ ${ }^{9}$ Department of Neuromedicine and Movement Science, Faculty of Medicine and Health Sciences, \\ NTNU Norwegian University of Science and Technology, Trondheim, Norway \\ ${ }^{10}$ Department of Otorhinolaryngology, Head and Neck Surgery, St. Olav's University Hospital, Trondheim, Norway
}

Correspondence should be addressed to Lars M. Berg; lars.m.berg@uit.no

Received 22 May 2021; Accepted 22 May 2021; Published 29 May 2021

Copyright (C) 2021 Lars M. Berg et al. This is an open access article distributed under the Creative Commons Attribution License, which permits unrestricted use, distribution, and reproduction in any medium, provided the original work is properly cited.

In the article titled "Health-Related Quality of Life and Sleep Quality after 12 Months of Treatment in Nonsevere Obstructive Sleep Apnea: A Randomized Clinical Trial with Continuous Positive Airway Pressure and Mandibular Advancement Splints" [1], the authors identified an error in Section 2.4 as follows:

"Hypopnea events were defined as $\geq 50 \%$ reduction in respiratory flow lasting $\geq 10 \mathrm{~s}$, with a simultaneous $\geq 3 \%$ reduction in peripheral blood oxygen saturation from baseline" should be corrected to "Hypopnea events were defined as $\geq 30 \%$ reduction in respiratory flow lasting $\geq 10 \mathrm{~s}$, with a simultaneous $\geq 3 \%$ reduction in peripheral blood oxygen saturation from baseline."

\section{References}

[1] L. M. Berg, T. K. S. Ankjell, Y.-Q. Sun et al., "Health-related quality of life and sleep quality after 12 months of treatment in nonsevere obstructive sleep apnea: a randomized clinical trial with continuous positive airway pressure and mandibular advancement splints," International Journal of Otolaryngology, vol. 2020, Article ID 2856460, 10 pages, 2020. 